\title{
The significance of the co-existence of osteopontin and tumor-associated macrophages in gastric cancer progression
}

\author{
Chang-Ni Lin ${ }^{1}$, Chih-Jung Wang ${ }^{2,3}$, Ying-Jui Chao ${ }^{2,3}$, Ming-Derg Lai ${ }^{1}$ and Yan-Shen Shan ${ }^{2,3^{*}}$
}

\begin{abstract}
Background: Osteopontin (OPN) can recruit macrophages to the site of inflammation and promote tumorigenesis. M2 tumor-associated macrophages (M2-TAMs) also play an important role in cancer progression. This study aimed to clarify the role of OPN and M2-TAMs co-existence in gastric cancer.

Methods: The levels of OPN and M2-TAMs were evaluated by immunohistochemical staining in 170 resected gastric cancer specimens that were collected from 1998 to 2012. M2-TAMs were identified by staining for an M2 marker, CD204. The prognostic significance and correlation between OPN and CD204 expression were analyzed. A co-culture system of OPN ${ }^{+}$-AGS and U937 cells was designed to study the effect of OPN on the skewing of macrophages toward M2-TAMs for gastric cancer progression in vitro and in vivo.
\end{abstract}

Results: Patients with high expression (>50\%) of OPN or CD204 exhibited poor 5-year overall survival rates (48.61\%, $p=0.0055$, and $52.14 \%, p=0.0498$, respectively). A positive correlation was observed between OPN and CD204 expression and high co-expression of OPN and CD204 demonstrated poor 5-year overall survival rates (48.90\%, $p=0.0131$ ). In the co-culture study, OPN was able to attract U937 cells and skew them toward M2-TAMs through paracrine action. The M2-TAMs could increase the invasiveness of OPN ${ }^{+}$-AGS cells and the growth rate of xenograft of a mixture of co-cultured OPN ${ }^{+}-\mathrm{AGS}$ and U937 cells.

Conclusion: OPN can skew macrophages toward M2-TAMs during gastric cancer progression. The co-existence of OPN and infiltrating M2-TAMs correlates with disease progression and poor survival and thus can serve as a prognostic marker in gastric cancer.

Keywords: Gastric cancer, Osteopontin, Tumor-associated macrophage, Biomarker, Cancer immunology

\section{Background}

Gastric cancer is the second leading cause of cancer death worldwide with overall 5 -year survival rates of less than 10\% [1]. The depth of invasion, extensive lymph node metastasis, and peritoneal seeding are the main reasons for high recurrence and mortality [2]. Surgery remains the only curative therapy for gastric cancer, although some studies reported that adjuvant chemotherapy and chemoradiation therapy can improve patient outcomes of resectable gastric cancers [3-5]. More than

\footnotetext{
* Correspondence: ysshan@mail.ncku.edu.tw

2Department of Surgery, National Cheng Kung University Hospital, College of Medicine, National Cheng Kung University, Tainan, Taiwan

${ }^{3}$ Institute of Clinical Medicine, College of Medicine, National Cheng Kung

University, Tainan, Taiwan

Full list of author information is available at the end of the article
}

$50 \%$ of gastric cancer patients who underwent radical resection ultimately suffered from local recurrence and distant metastasis [6]. Therefore, a comprehensive investigation of the molecular mechanisms underlying the development and progression of gastric cancer is critical for designing better therapeutic strategies for the treatment of gastric cancer.

Epidemiological studies reported that chronic inflammation predisposes cells to malignancy. Additionally, inhibition of chronic inflammation in patients with premalignant disease could reduce cancer risk and cancer recurrence [7], suggesting that chronic inflammation can generate a beneficial microenvironment for tumor progression and metastatic dissemination. Previous studies reported that gastric cancer is often accompanied by the 
phenomena of gastritis. Gastric adenocarcinoma was also found to frequently occur in areas of chronic inflammation $[8,9]$. Furthermore, gastritis appears to be closely associated with an increased risk of developing gastric cancer [10]. Therefore, chronic inflammation is believed to be an important factor in driving gastric cancer progression.

The tumor microenvironment is a complex milieu that comprises various inflammatory cells and a network of signaling molecules. Among the inflammatory cells, the aberrant infiltration and activation of macrophages is frequently observed in gastric inflammation and cancer [11]. The infiltrating macrophages, also termed tumorassociated macrophages (TAMs), are associated with poor prognosis in a variety of human cancers and play important roles in tumor development [12]. In gastric carcinomas, TAMs infiltration in tumors is associated with more malignant phenotypes, including tumor angiogenesis, depth of invasion, nodal status, and clinical stages $[13,14]$. Gastric cancer patients with a high level of TAMs infiltration demonstrated worse outcomes after surgery than those with a low level of TAMs infiltration [14]. However, the precise role of TAMs in gastric cancer remains unknown.

Osteopontin (OPN) is a secreted matrix glycoprotein that regulates a number of biological processes. The overexpression of OPN was observed in various human cancers and is associated with poor patient outcomes in a variety of cancers, such as breast cancer [15], lung cancer [16], liver cancer [17], gastric cancer [18], colon cancer [19], and cervical cancer [20]. In gastric cancer, OPN has been reported to promote cell growth, invasion, and metastasis, whereas knockdown of OPN attenuated these effects in vitro and in vivo [21,22]. We therefore sought to clarify the correlation between OPN and TAMs in gastric cancer and its clinical significance.

\section{Methods}

Immunohistochemistry

For immunohistochemistry, paraffin embedded samples of 170 gastric cancer patients who underwent potentially curative surgery between 1998 and 2012 at the National Cheng Kung University Hospital (NCKUH), Tainan, Taiwan were immunostained with an anti-human OPN antibody (1:200; ab8448; Abcam) and an anti-human CD204 antibody, a marker of M2 type tumor associated macrophage (M2-TAM) (1:200; ab53566; Abcam). After horseradish peroxidase (HRP)-conjugated IgG was added for 1 hour, the specimens were analyzed by $A B C$ detection. The degrees of staining intensity were classified into four grades by comparison with the controls as follows: 0 , negative (same as the negative control); $\mathrm{A}$, weak staining $(<25 \%$ of the area); B, moderate staining $(\geq 25 \%$ but $<50 \%$ of the area); and C, extensive staining ( $>50 \%$ of the area). Grade $\mathrm{C}$ was considered to represent high expression of the stained protein. This study was approved by Human Experimental and Ethics Committee of National Cheng Kung University Hospital (ER-98017). The written informed consent for participation in the study was obtained from participants.

The xenografts samples were immunostained with an anti-mouse CD31 antibody (1:200; 550274; BD Pharmingen) and an anti-mouse $\alpha$-smooth muscle actin ( $\alpha$-SMA) antibody (1:50; ab5694; Abcam). The secondary antibodies, including HRP-conjugated IgG and fluorophoreconjugated IgG, were selected for imaging.

\section{Co-culture method for studying paracrine effect}

Cell lines including monocyte cell line U937, TAM primary cultured from gastric cancer specimens $\left(\mathrm{TAM}^{\mathrm{cli}}\right.$ ),

Table 1 The clinicopathological characteristics of 170 patients with gastric cancer

\begin{tabular}{|c|c|c|}
\hline & Number (N) & Percentage (\%) \\
\hline \multicolumn{3}{|l|}{ Gender } \\
\hline Male & 97 & 57.06 \\
\hline Female & 73 & 42.94 \\
\hline \multicolumn{3}{|l|}{ Age } \\
\hline$\leq 50$ years & 34 & 20.00 \\
\hline$>50$ years & 136 & 80.00 \\
\hline \multicolumn{3}{|c|}{ Tumor Location } \\
\hline Proximal & 79 & 46.47 \\
\hline Distal & 91 & 53.53 \\
\hline \multicolumn{3}{|c|}{ Tumor Size (cm) } \\
\hline$\leq 5$ & 115 & 67.65 \\
\hline$>5$ & 55 & 32.35 \\
\hline \multicolumn{3}{|c|}{ Lauren Classification } \\
\hline Intestinal & 73 & 42.94 \\
\hline Diffuse & 97 & 57.06 \\
\hline \multicolumn{3}{|c|}{ Tumor Stage } \\
\hline Early & 90 & 52.94 \\
\hline Advanced & 80 & 47.06 \\
\hline \multicolumn{3}{|c|}{ LN Metastasis } \\
\hline No & 74 & 43.53 \\
\hline Yes & 96 & 56.47 \\
\hline \multicolumn{3}{|c|}{ Endpoint Status } \\
\hline Survival & 95 & 55.88 \\
\hline Death & 75 & 44.12 \\
\hline \multicolumn{3}{|c|}{ OPN Staining } \\
\hline$\leq 50 \%$ & 79 & 46.47 \\
\hline$>50 \%$ & 91 & 53.53 \\
\hline \multicolumn{3}{|c|}{ CD204 Staining } \\
\hline$\leq 50 \%$ & 79 & 46.47 \\
\hline$>50 \%$ & 91 & 53.53 \\
\hline
\end{tabular}


gastric cancer cell line AGS with expression of OPN $\left(\mathrm{OPN}^{+}\right.$-AGS), and AGS with knockdown of OPN by short hairpin RNA (OPN-shRNA AGS) were used for co-culture in this study. A Boyden chamber with a 4-nm pore size insert was used for co-culture. The U937 or $\mathrm{TAM}^{\mathrm{cli}}$ were seeded inside in the insert, and $\mathrm{OPN}^{+}$-AGS or OPN-shRNA AGS were seeded in the base of the chamber. After incubation for 72 hours, the condition medium was collected for future chemoattractant experiments. A monoclonal antibody against OPN, or recombinant OPN (rOPN) was also used to observe the chemoattractant effects of OPN during incubation for 72 hours.

\section{Invasion assay}

After the co-culture treatment, $5 \times 10^{5}$ gastric cancer cells were moved onto $8-\mu \mathrm{m}$ pore polycarbonate inserts containing Matrigel (354234; BD Pharmingen) and incubated at $37^{\circ} \mathrm{C}$. After 24 hours, the membrane was torn off slowly, washed in PBS, and stained with Giemsa. These invasive cells were counted under microscopy and photographed.

\section{Animal model}

Four- to 6-week-old nude mice were obtained from the National Laboratory Animal Center. The housing and experimental animal procedures were approved by the Institutional Animal Care and Use Committee of NCKU (IACUC 98219). Gastric cancer cells $\left(1 \times 10^{6}\right)$ were intradermally injected into the nude mice either alone or mixed with U937 $\left(1 \times 10^{6}\right)$ after a 72-hour co-culture. The xenografts were observed for 9 weeks until the mice were sacrificed and were paraffin-embedded for histological analysis.

\section{Statistical analysis}

Univariate and multivariate analysis were used to compare the overall survival and the pathology variables. Receiver operating characteristic (ROC) curve analysis was used to determine the value demonstrating the highest accuracy in predicting patient outcomes. The prognostic assessment was performed by Kaplan-Meier survival and Cox Regression analysis to identify significance. Chi-squared tests were used to analyze the correlation between OPN/CD204 staining and the clinical pathologic features. The relationship between the two variables of OPN and CD204 was analyzed by a one-way ANOVA test in GraphPad Prism 5.0 software, and most of analysis was calculated by SPSS 17.0. Statistical significance was set at $p<0.05$.

\section{Results}

OPN and CD204 were highly expressed in gastric tumor and correlated with disease progression

The clinicopathological characteristics of the gastric cancer patients were described in Table 1. Males constituted $57.06 \%(97 / 170)$ of the patient population, and $46.47 \%$ $(79 / 170)$ of cases were proximal gastric cancers. Most of

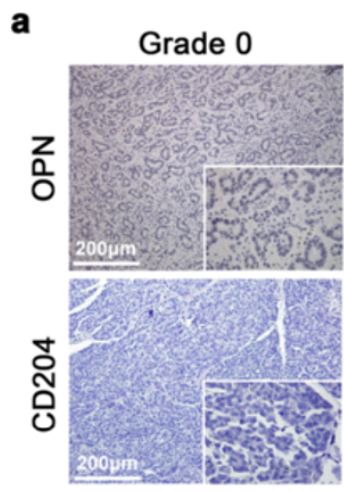

b

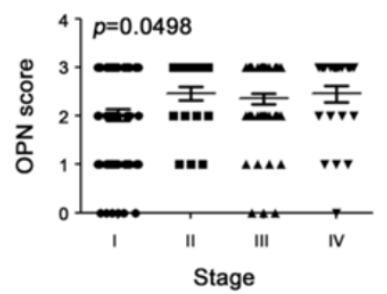

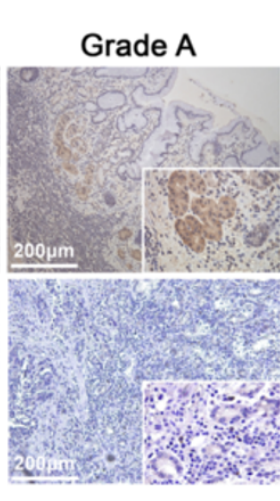

C

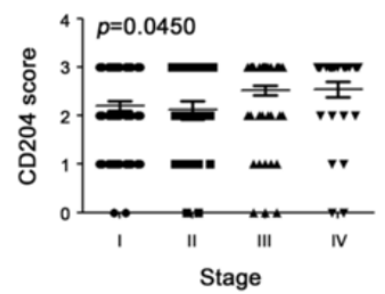

Grade B

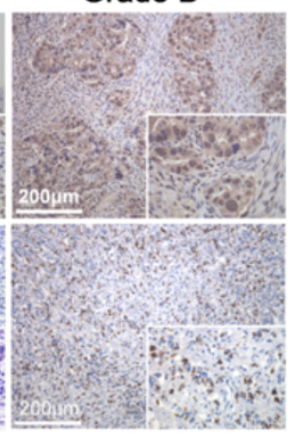

d

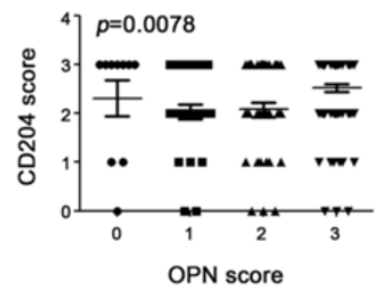

Figure 1 The existence of OPN and M2-TAMs in gastric cancer specimens; the M2-TAMs were stained with a CD204-specific antibody. (a) The stain intensity is shown from grade 0 to grade C. (b, c) The expression of OPN and CD204 was significantly correlated with the tumor stage $(p<0.05)$. (d) The OPN score was positively correlated with the CD204 score $(p=0.0078)$. 
the cases were early gastric cancer $(52.94 \%, 90 / 170)$. The mean follow-up time was 42.2 months (median followup time was 28.5 months). The different grading of OPN and CD204 expression in the gastric cancer specimens were shown in Figure 1a. A total of 53.53\% of the cancer tissue samples demonstrated high expression of both OPN and CD204. The expression scores of OPN and CD204 were correlated with the tumor stage, $p=0.0498$ and $p=0.0450$, respectively (Figure $1 \mathrm{~b}-\mathrm{c}$ ). Furthermore, a positive correlation was observed between OPN and
CD204 expression in gastric cancer, $R$ square $\left(R^{2}\right)$ is 0.0630 and $p=0.0078$ (Figure 1d). The results indicated that M2-TAMs infiltration in gastric cancer tissue was correlated with OPN expression as the disease progressed.

\section{Co-expression of OPN and CD204 was significantly associated with overall survival}

In univariate and multivariate analysis, the tumor size, tumor stage, lymph node metastasis, OPN expression, and CD204 expression were significantly associated with overall

Table $\mathbf{2}$ The overall survival of $\mathbf{1 7 0}$ gastric cancer patients is analyzed after resection

\begin{tabular}{|c|c|c|c|c|c|c|c|}
\hline & \multicolumn{3}{|c|}{ Univariate analysis $(\mathrm{N}=170)$} & \multicolumn{4}{|c|}{ Multivariate analysis $(\mathrm{N}=170)$} \\
\hline & \multicolumn{3}{|c|}{ Endpoint status (\%) } & \multirow[b]{2}{*}{ Mean } & \multicolumn{2}{|c|}{$95 \% \mathrm{Cl}$} & \multirow[b]{2}{*}{$p$ value } \\
\hline & Survival & Death & $p$ value & & Lower & Upper & \\
\hline Gender & & & 0.317 & 1.425 & 1.349 & 1.501 & 0.320 \\
\hline Male & $51(53.7)$ & $44(46.3)$ & & & & & \\
\hline Female & $46(61.3)$ & $29(38.7)$ & & & & & \\
\hline Age & & & 0.440 & 64.797 & 62.673 & 66.92 & 0.718 \\
\hline$\leq 50$ years & $17(50.0)$ & $17(50.0)$ & & & & & \\
\hline$>50$ years & $78(57.4)$ & $58(43.6)$ & & & & & \\
\hline Tumor Location & & & 0.566 & 2.494 & 2.372 & 2.617 & 0.991 \\
\hline Proximal & $46(58.2)$ & $33(41.8)$ & & & & & \\
\hline Distal & $49(53.8)$ & $42(46.2)$ & & & & & \\
\hline Tumor Size (cm) & & & 0.011 & 4.632 & 4.208 & 5.507 & 0.006 \\
\hline$\leq 5$ & $72(62.6)$ & $43(37.4)$ & & & & & \\
\hline$>5$ & $23(41.8)$ & $32(58.2)$ & & & & & \\
\hline Lauren Classification & & & 0.189 & 2.016 & 1.875 & 2.158 & 0.212 \\
\hline Intestinal & $45(61.6)$ & $28(38.4)$ & & & & & \\
\hline Diffuse & $50(51.5)$ & $47(48.5)$ & & & & & \\
\hline Tumor Stage & & & 0.000 & 1.491 & 1.419 & 1.563 & 0.000 \\
\hline Early & $65(68.4)$ & $25(33.3)$ & & & & & \\
\hline Advance & $30(31.6)$ & $50(66.7)$ & & & & & \\
\hline LN Metastasis & & & 0.000 & 0.584 & 0.512 & 0.656 & 0.000 \\
\hline No & $55(74.3)$ & $19(25.7)$ & & & & & \\
\hline Yes & $40(41.7)$ & $56(58.3)$ & & & & & \\
\hline OPN Staining & & & 0.015 & 2.266 & 2.125 & 2.407 & 0.026 \\
\hline$\leq 50 \%$ & $52(65.8)$ & $27(34.2)$ & & & & & \\
\hline$>50 \%$ & $43(47.3)$ & $48(52.7)$ & & & & & \\
\hline CD204 Staining & & & 0.034 & 2.304 & 2.169 & 2.439 & 0.049 \\
\hline$\leq 50 \%$ & 51 & 28 & & & & & \\
\hline$>50 \%$ & 44 & 47 & & & & & \\
\hline OPN vs. CD204 Staining & & & 0.007 & 2.678 & 2.494 & 2.861 & 0.001 \\
\hline$\leq 50 \%$ vs. $\leq 50 \%$ & $36(76.6)$ & $11(23.4)$ & & & & & \\
\hline$\leq 50 \%$ vs. $>50 \%$ & $16(53.3)$ & $14(46.7)$ & & & & & \\
\hline$>50 \%$ vs. $\leq 50 \%$ & $15(50.0)$ & $15(50.0)$ & & & & & \\
\hline$>50 \%$ vs. $>50 \%$ & $28(44.4)$ & $35(55.6)$ & & & & & \\
\hline
\end{tabular}


survival of patients with gastric cancer (Table 2). Notably, the co-expression of OPN and CD204 was more significantly associated with overall survival $(p<0.01)$ compared with OPN $(p<0.05)$ or CD204 $(p<0.05)$ alone. To confirm the correlation between the co-expression of OPN and CD204 and overall survival, ROC curve analysis was used. We found that the co-expression of OPN and CD204 was highly associated with overall survival. The tumor size $(p=0.005)$, tumor stage $(p=0.000)$, lymph node metastasis $(p=0.000)$, and co-expression of OPN and CD204 $(p=0.002)$ were significantly associated with the overall outcome (Figure 2). The results demonstrated that the co-existence of OPN and M2-TAMs in gastric cancer was highly associated with the overall survival of gastric cancer patients.

\section{High co-expression of OPN and CD204 was a marker of poor prognosis}

Next, we used the chi-square test to evaluate the correlation between OPN/CD204 expression and clinicopathologic characteristics (Table 3). We noted that the tumor size $(p=0.031)$, endpoint status $(p=0.015)$, and CD204 expression $(p<0.001)$ were significantly correlated with OPN expression. In the CD204 analysis results, the tumor stage $(p=0.006)$, endpoint status $(p=0.034)$, and OPN expression $(p<0.001)$ were also significantly correlated. Interestingly, OPN expression was significantly correlated with CD204 expression in gastric cancer. In conjunction with the results shown in Figure 1d, M2-TAMs infiltration in gastric tumors was highly correlated with OPN expression. Thus, we next analyzed whether co-expression of OPN and CD204 correlated with clinicopathologic characteristics. Similarly, the tumor stage $(p=0.004)$ and endpoint status $(p=0.007)$ were significantly correlated with the co-expression of OPN and CD204. The results prove that the coexpression of OPN and CD204 was associated with the tumor stage and predicted worse patient outcomes.

Kaplan-Meier survival analysis was used to determine the overall survival of patients with gastric cancer (Figure 3). Patients with high expression of OPN demonstrated significantly worse overall survival than those with low expression of OPN $(p=0.0055)$ and the hazard ratio is 2.039 (95\% CI of ratio is 1.220 to 3.406 ). The 5 -year survival rate of gastric cancer patients with high expression OPN was $48.61 \%$, whereas for patients with low expression OPN the 5-year survival rate was $70.42 \%$. In M2-TAMs analysis, we found that patients with high expression of CD204 exhibited lower overall survival $(\mathrm{p}=0.0498)$, hazard ratio is 1.653 (95\% CI of ratio is 0.995 to 2.745 ) and a lower 5-year survival rate (52.14\%), compared with low CD204 expressing patients. Furthermore, the 5-year survival rate of patients with high co-expression of OPN and CD204 was 48.90\%, whereas that of patients with low co-expression of OPN and CD204 was $82.10 \%$. These results suggest that high co-expression of OPN and CD204 was a marker of poor prognosis in gastric cancer.

\section{A paracrine regulation between OPN and M2-TAMs in gastric cancer}

The dual immunofluorescence results indicated that OPN and CD204 were co-localized even in high-expression or

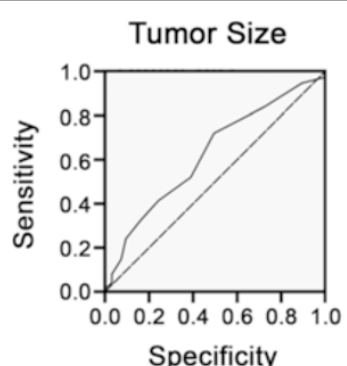

Specificity

OPN and CD204

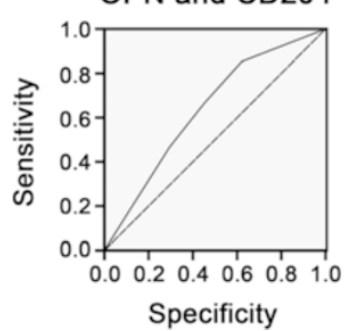

Tumor Stage

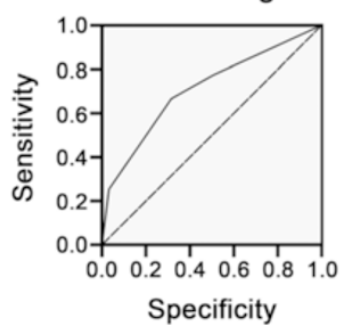

Specificity

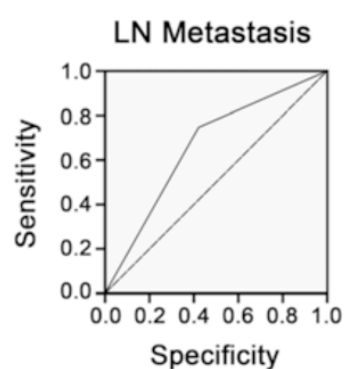

\begin{tabular}{|l|cccccc|}
\hline & & & & & \multicolumn{2}{c|}{$95 \% \mathrm{Cl}$} \\
\cline { 5 - 7 } & Area under ROC curve & SD & $p$ value & lower & upper \\
\hline Tumor Size & 0.624 & 0.043 & 0.005 & 0.540 & 0.709 \\
Tumor Stage & 0.710 & 0.041 & 0.000 & 0.630 & 0.789 \\
LN Metastasis & 0.663 & 0.042 & 0.000 & 0.581 & 0.745 \\
OPN and CD204 & 0.637 & 0.042 & 0.002 & 0.554 & 0.720 \\
\hline
\end{tabular}

Figure 2 Receiver operating characteristic (ROC) curves for the tumor size, tumor stage, lymph node metastasis, and co-expression of OPN and CD204 were used to predict overall gastric cancer survival. The area under the ROC curve for tumor size was $0.624(95 \% \mathrm{Cl}$, 0.540-0.709), tumor stage was 0.710 (95\% Cl, 0.630-0.789), lymph node metastasis was 0.663 (95\% Cl, 0.581-0.745), and co-expression of OPN and CD204 was 0.637 (95\% Cl, 0.554-0.720). These parameters were significantly associated with overall survival $(p=0.005,0.000,0.000$, and 0.002$)$. 
Table 3 OPN and CD204 expression correlated with clinicopathologic characterization

\begin{tabular}{|c|c|c|c|c|c|c|c|c|c|c|c|}
\hline & \multicolumn{3}{|c|}{ OPN expression (\%) } & \multicolumn{3}{|c|}{ CD204 expression (\%) } & \multicolumn{5}{|c|}{ OPN vs. CD204 expression (\%) } \\
\hline & $\leq 50 \%$ & $>50 \%$ & $\overline{p \text { value }}$ & $\leq 50 \%$ & $>50 \%$ & $p$ value & $\begin{array}{l}\leq 50 \% \text { vs. } \\
\leq 50 \%\end{array}$ & $\leq 50 \%$ vs. $>50 \%$ & $\begin{array}{l}>50 \% \text { vs. } \\
\leq 50 \%\end{array}$ & $\begin{array}{l}>50 \% \text { vs. } \\
>50 \%\end{array}$ & $p$ value \\
\hline Gender & & & 0.550 & & & 0.981 & & & & & 0.795 \\
\hline Male & $47(59.5)$ & $50(54.9)$ & & $45(57.0)$ & $52(57.1)$ & & $29(61.7)$ & $17(56.7)$ & $15(50.0)$ & $36(57.1)$ & \\
\hline Female & $32(40.5)$ & $41(45.1)$ & & $34(43.0)$ & $39(42.9)$ & & 18 (38.3) & $13(43.3)$ & $15(50.0)$ & $27(42.9)$ & \\
\hline Age & & & 0.758 & & & 0.489 & & & & & 0.606 \\
\hline$\leq 50$ years & $15(19.0)$ & $19(20.9)$ & & $14(17.7)$ & $20(22.0)$ & & $7(14.9)$ & $8(26.7)$ & $7(23.3)$ & $12(19.0)$ & \\
\hline$>50$ years & $64(81.0)$ & $72(79.1)$ & & $65(82.3)$ & $71(78.0)$ & & $40(85.1)$ & $22(73.3)$ & $23(76.7)$ & $51(81.0)$ & \\
\hline Tumor Location & & & 0.146 & & & 0.480 & & & & & 0.229 \\
\hline Proximal & $32(45.5)$ & $47(51.6)$ & & $39(49.4)$ & $40(44.0)$ & & $22(46.8)$ & $9(30.0)$ & $16(53.3)$ & $32(50.8)$ & \\
\hline Distal & $47(59.5)$ & $44(48.4)$ & & $40(50.6)$ & $51(56.0)$ & & $25(53.2)$ & $21(70.0)$ & $14(46.7)$ & $31(49.2)$ & \\
\hline Tumor Size $(\mathrm{cm})$ & & & 0.031 & & & 0.068 & & & & & 0.135 \\
\hline$\leq 5$ & $60(75.9)$ & $55(24.1)$ & & $59(74.7)$ & $56(61.5)$ & & $38(80.9)$ & $20(66.7)$ & 19 (63.3) & $38(60.3)$ & \\
\hline$>5$ & 19 (60.4) & 36 (39.6) & & $20(25.3)$ & 35 (38.5) & & $25(19.1)$ & 21 (33.3) & $14(36.7)$ & $31(39.7)$ & \\
\hline Lauren Classificat & & & 0.519 & & & 0.738 & & & & & 0.983 \\
\hline Intestinal & $36(45.6)$ & $37(40.7)$ & & $35(44.3)$ & $38(41.8)$ & & $21(44.7)$ & $13(43.3)$ & $12(40.0)$ & $27(42.9)$ & \\
\hline Diffuse & $43(54.4)$ & $54(59.3)$ & & $44(55.7)$ & $53(58.2)$ & & $26(55.3)$ & $17(56.7)$ & $18(60.0)$ & $36(51.1)$ & \\
\hline Tumor Stage & & & 0.379 & & & 0.006 & & & & & 0.004 \\
\hline Early & $46(58.2)$ & $44(48.4)$ & & $53(67.1)$ & $37(40.7)$ & & $34(72.3)$ & $11(36.7)$ & $18(60.0)$ & $27(42.9)$ & \\
\hline Advance & $33(41.8)$ & $47(51.6)$ & & $26(32.9)$ & $54(59.3)$ & & $13(27.7)$ & $19(63.3)$ & $12(40.0)$ & $36(57.1)$ & \\
\hline LN Metastasis & & & 0.263 & & & 0.082 & & & & & 0.345 \\
\hline No & $38(48.1)$ & 36 (39.6) & & $40(50.6)$ & $34(37.4)$ & & $25(53.2)$ & $12(40.0)$ & $14(46.7)$ & $23(36.5)$ & \\
\hline Yes & $41(51.9)$ & $55(60.4)$ & & $39(49.4)$ & $57(62.6)$ & & $22(46.8)$ & $18(60.0)$ & $16(53.3)$ & $40(63.5)$ & \\
\hline Endpoint Status & & & 0.015 & & & 0.034 & & & & & 0.007 \\
\hline Survival & $52(65.8)$ & $43(47.3)$ & & $51(64.6)$ & $44(48.4)$ & & $36(76.6)$ & $16(53.3)$ & $15(50.0)$ & $28(44.4)$ & \\
\hline Death & $27(34.2)$ & $48(52.7)$ & & $28(35.4)$ & 47 (51.6) & & $11(23.4)$ & $14(46.7)$ & $15(50.0)$ & 35 (55.6) & \\
\hline
\end{tabular}

Statistically significant $p$ values are shown in bold; $p$ values were calculated using Fisher's exact test.

low-expression gastric cancer specimens. This finding suggests that OPN was bound to the surface of M2TAMs. The IHC staining demonstrated that the macrophages were located beside the tumor cells (Figure 4a). The results implied that paracrine regulation occurs between OPN and M2-TAMs within gastric cancer. Thus, we designed a co-culture system to mimic the tumor microenvironment. To explore the chemoattractant effect of OPN on M2-TAMs infiltration, U937 $\left(5 \times 10^{5}\right)$ were cultured in the insert, and $\mathrm{OPN}^{+}$-AGS gastric cancer cells were grown on the base of a Boyden chamber (Figure 4b). After incubated for 72 hours, the condition medium was collected and added into another lower chamber. New insert containing U937 cells was put into the chamber and then incubated for 72 hours. The number of M2-TAMs in the lower chamber was significantly increased, compared with those cells treated with conditioning medium containing a monoclonal OPN antibody (Figure 4c). Furthermore, when rOPN was added to the normal medium, M2-TAMs were still observed in the base of chamber. Flow cytometry further proved that the M2-TAMs differentiation was associated with the presence of OPN (Figure $4 \mathrm{~d}$ ). Taken together, these results suggest that OPN was required for M2-TAMs infiltration in gastric cancer.

After being co-cultured with $\mathrm{OPN}^{+}$-AGS cells, the U937 cell demonstrated significantly increased mRNA levels of CD204 and IL-10, as in TAM ${ }^{\text {cli }}$ cells, when compared with cells co-cultured with $O P N$-shRNA AGS cells, stimulated with LPS (to become M1 macrophages exhibiting increased mRNA levels of IL-1) or treated with an OPN monoclonal antibody (Figure 5a). This result proved that OPN was able to recruit monocytes and skewed them toward a M2-TAMs phenotype in gastric cancer microenvironments.

\section{M2-TAM promoted gastric cancer progression}

After being co-cultured with U937 or TAM ${ }^{\text {cli }}$ cells for 3 days, the invasiveness of $\mathrm{OPN}^{+}$-AGS cells was significantly increased, but the invasiveness was reduced after the addition of OPN antibodies (Figure 5b). To assess 


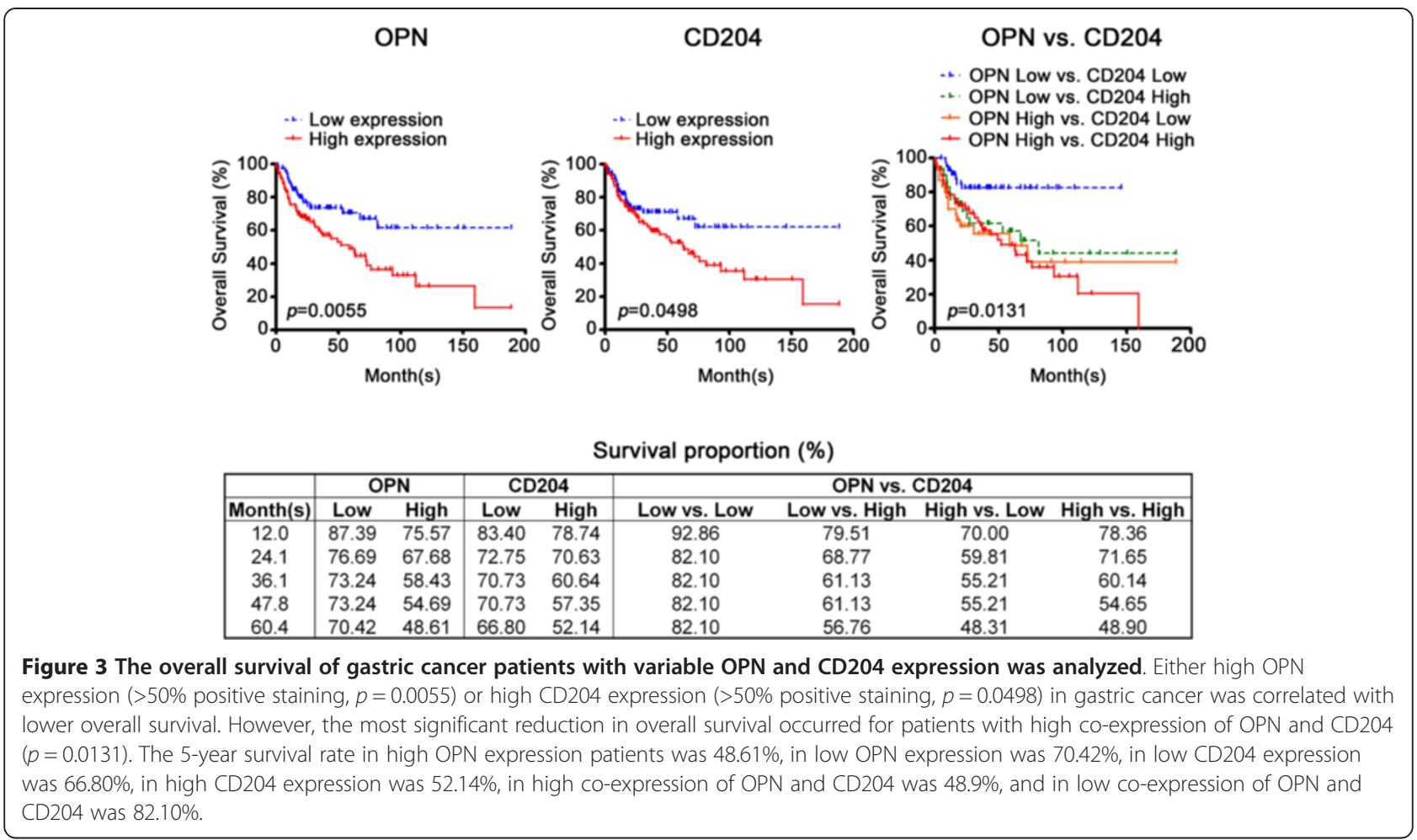
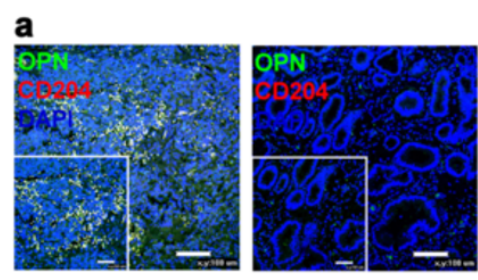

c

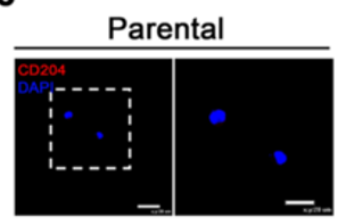

OPN ab

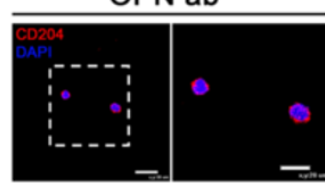

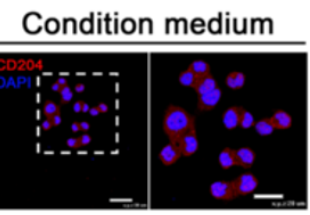

rOPN

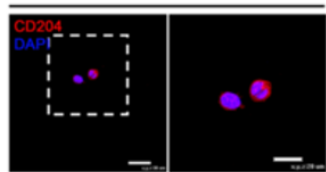

b
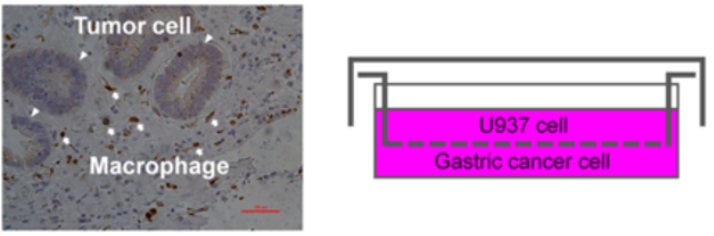

d

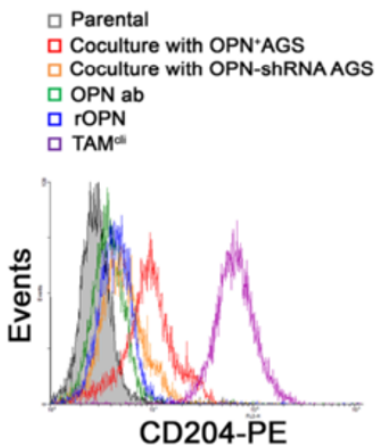

Figure 4 The paracrine effect of OPN skewed macrophages toward M2-TAMs in gastric cancer. (a) Dual immunofluorescence staining of OPN (green) and CD204 (red) shows co-localization (yellow) in gastric cancer specimens. The immunohistochemistry of CD204 shows that macrophages did not come in contact with tumor cells. (b) A co-culture method was designed to mimic the tumor microenvironment of gastric cancer without direct contact between cancer cells and macrophages. (c) The confocal images show that OPN contributed to the recruitment of U937 cells and skewed the cells toward M2-TAMs after treatment with conditioning medium; this phenomenon could be blocked by an OPN monoclonal antibody. Recombinant OPN was able to increase CD204 expression on macrophages. (d) The amount of CD204 ${ }^{+}$TAMs was shown by flow cytometry. The CD204+-TAM phenotype increased dramatically after co-culture with OPN ${ }^{+}$AGS cells (red, open histogram). Parental cells (filled histogram), OPN-shRNA AGS cells (orange, open histogram), OPN neutralizing antibody (green, open histogram), or recombinant OPN (blue, open histogram) were compared. The TAM cli cells isolated from human gastric cancer were used as a positive control. 

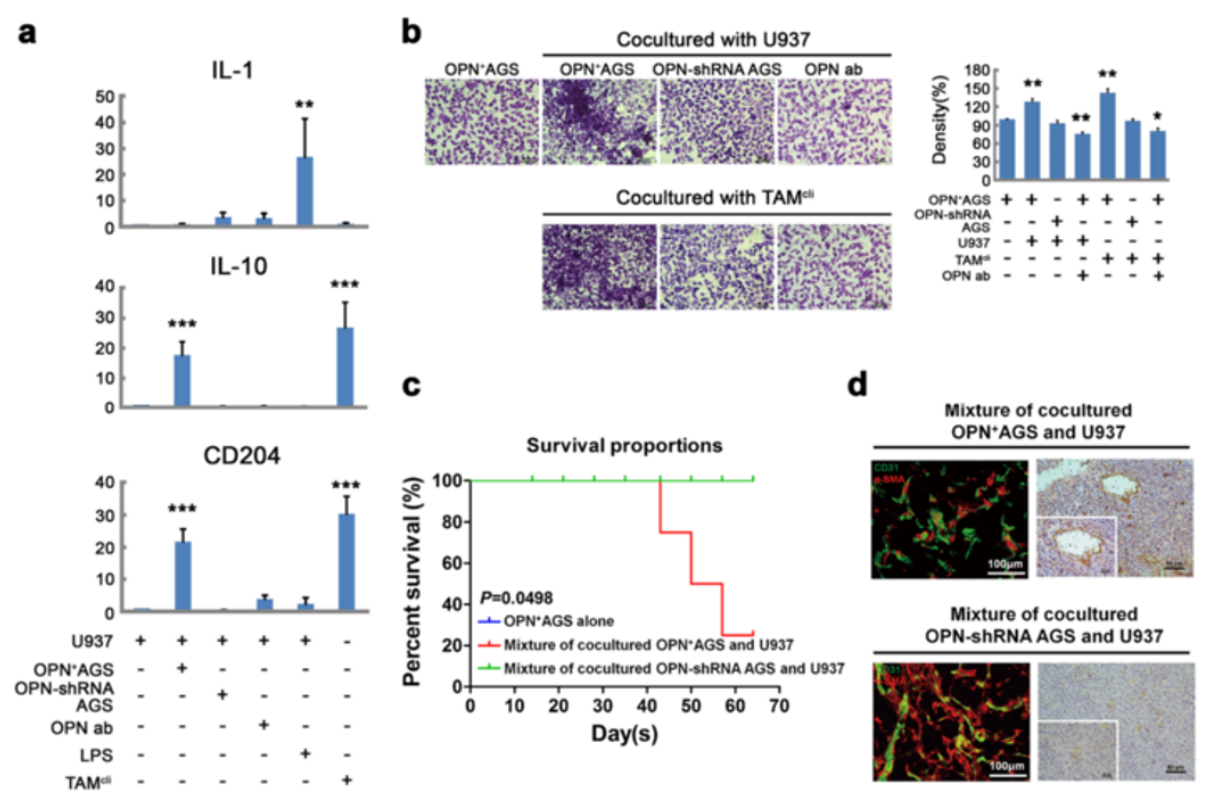

Figure 5 The co-expression of OPN and M2-TAMs promotes gastric tumorigenesis. (a) The expression of M1/M2 markers in U937 cells was analyzed after co-culture treatment by real-time PCR. LPS-treated U937 cells expressed IL-1 as M1 macrophages, and U937 cells alone were used as a negative control. TAM cli cells isolated from clinical specimens were used as a positive control. After co-culture with OPN ${ }^{+}$-AGS cells, the U937 cells differentiated into M2-TAMs expressing high levels of CD204 and IL-10. (b) After 72 hours of co-culture with U937 or TAM cli cells, the invasiveness of the OPN ${ }^{+}$-AGS cells increased but that of OPN-shRNA AGS cells did not. The increased cell invasiveness was also blocked by an OPN monoclonal antibody. (c) The mixture of co-cultured OPN+-AGS and U937 cells inoculated into nude mice showed poor survival compared with a mixture of co-cultured OPN-shRNA AGS and U937 cells or OPN ${ }^{+}$-AGS cells alone $(p=0.0498)$. (d) The neovascularization in the xenografts was shown by double-staining with anti-CD31 and anti-aSMA antibodies. Less neovascularization with normal vascular structure was found in xenografts of a mixture of co-cultured OPN-shRNA AGS and U937 cells.

the effects of TAMs on tumorigenesis in vivo, a mixture of co-cultured OPN ${ }^{+}$-AGS and U937 cells was inoculated into the back skin of nude mice to observe the growth of xenografts. Compared with inoculation of $\mathrm{OPN}^{+}$-AGS cells alone, the xenografts from mixture of co-cultured $\mathrm{OPN}^{+}$-AGS and U937 cells grew faster and disseminated to the liver and peritoneal cavity, similar to human gastric cancer. Those nude mice exhibited poor survival compared with mice inoculated with a mixture of OPNshRNA and U937 cells or AGS cells alone (Figure 5c). We also found that a marked neovascularization occurred in the xenografts from a mixture of co-cultured $\mathrm{OPN}^{+}$-AGS and U937 cells, but the neovascularization was reduced in the tumors generated from a mixture of OPN-shRNA AGS and U937 cells (Figure 5d). Collectively, these results proved that OPN could recruit macrophages and skew them toward M2-TAMs formation and the M2-TAMs further promoted gastric cancer progression.

\section{Discussion}

Recently, many studies have proven that the infiltrating inflammatory cells in the tumor microenvironment could promote cancer progression [23,24]. Chronic inflammation is frequently found within gastric tumors, and M2-TAMs can be observed after staining for M2 markers. The correlation between the presence of M2TAMs in tumors and poor survival has been demonstrated in several cancer types [25-27]. However, the reasons underlying the ability of infiltrating TAMs to promote cancer progression remain a mystery. We observed that OPN was highly expressed in gastric cancer specimens and positively correlated with M2-TAMs infiltration $\left(R^{2}=0.7743\right)$. More importantly, the coexistence of OPN and M2-TAMs is significantly correlated with poor prognosis and lower 5-year survival rates. Although our results are similar to those of previous reports, we focused on M2-type macrophages (CD204 staining) rather than total macrophages (CD68 staining) $[28,29]$. In addition, a previous study demonstrated that OPN overexpression in TAMs was able to enhance angiogenesis and growth in melanoma through autocrine signaling [30] rather than through the interactions with tumor cells. Therefore, this is the first study to prove the paracrine regulation of M2-TAMs by OPN to promote gastric cancer progression.

OPN is a secreted glycoprotein that can generate macrophage accumulation [31] and enhance tumor invasion $[32,33]$. However, the detailed mechanism remains unclear. In this study, we used a co-culture system to demonstrate a paracrine regulation between OPN and 
M2-TAMs in gastric cancer. OPN has the ability to recruit and skew macrophages toward M2-TAMs and thus promotes gastric cancer progression. OPN has been reported to promote invasion and metastasis of gastric cancer through HIF- $1 \alpha$ upregulation and MMP9 activation [34]. Moreover, the plasma OPN concentration in patients with metastatic disease is significantly higher than that in patients without metastases [35]. In breast cancer, OPN can promote cancer progression, whereas knockdown of OPN aborts this effect [36]. Notably, contradictory results were observed in an $O P N$ knockout squamous carcinoma mouse model. Primary skin tumors grew larger and produced more numerous lung metastases in $O P N$-deficient mice, compared with their wildtype counterparts [37]. The controversial findings may result from the different functions of OPN in normal tissues and tumors. Our results are consistent with a previous study which reported that the tumor microenvironment determines the effects of OPN [38]. In the future, we will further clarify the mechanism underlying the interaction between OPN and TAMs in gastric cancer.

\section{Conclusion}

Our study clearly demonstrates that clinical parameters, including tumor size, tumor stage, lymph node metastasis, OPN expression, and TAMs infiltration are associated with overall survival of gastric cancer patients. Patients with high co-expression of OPN and CD204 exhibit a lower 5-year survival rate. In vitro and in vivo experiments further verify the interaction between OPN and TAMs, which can promote gastric cancer progression. Our novel findings provide a good marker for predicting the outcomes of patients with gastric cancer.

\begin{abstract}
Abbreviations
TAM: Tumor associated macrophage; M2-TAM: M2 type tumor associated macrophage; OPN: Osteopontin; a-SMA: a-smooth muscle actin; HRP: Horseradish peroxidase; IgG: Immunoglobulin G; shRNA: Short hairpin RNA; ROC: Receiver operating characteristic; NCKUH: National Cheng Kung University Hospital; IACUC: Institutional Animal Care and Use Committee; IOPN: Recombinant OPN; TAM cli: Human TAM from gastric cancer specimens; $\mathrm{OPN}^{+}$-AGS: Gastric cancer cell line AGS with expression of OPN; OPN-shRNA
\end{abstract} AGS: AGS with knockdown of OPN by short hairpin RNA.

\section{Competing interests}

The authors declare that they have no competing interests.

\section{Authors' contributions}

CNL carried out most of the studies, performed the statistical analysis and drafted the manuscript. CJW, YJC and YSS performed the operation and collected clinical data. M-DL participated in the design of the study. YSS got the grant for the study, designed and conceived of the study, and participated in coordination and helped to draft and revise the manuscript. All authors read and approved the final manuscript.

\section{Authors' information}

Chang-Ni Lin is a graduated student. Ying-Jui Chao and Chih-Jung Wang are attending surgeons and graduated students under instruction by Yan-Shen Shan. Ming-Derg Lai is the chief of Institute of Basic Medical Sciences, College of Medicine, NCKU, Tainan, Taiwan; Yan-Shen Shan is a professor of
Institute of Clinical Medicine, College of Medicine, NCKU, Tainan, Taiwan, and an attending surgeon of Department of Surgery, NCKUH, Tainan, Taiwan.

\section{Acknowledgement}

This study was funded from the Department of Health, Executive Yuan, Taiwan (DOH101-TD-C-111-003), the National Cheng Kung University Hospital (NCKUH-9801005), and the National Science Council, Taiwan (NSC99-2314-B-006-020-MY2).

\section{Author details}

'Institute of Basic Medical Sciences, College of Medicine, National Cheng Kung University, Tainan, Taiwan. ${ }^{2}$ Department of Surgery, National Cheng Kung University Hospital, College of Medicine, National Cheng Kung University, Tainan, Taiwan. ${ }^{3}$ Institute of Clinical Medicine, College of Medicine, National Cheng Kung University, Tainan, Taiwan.

Received: 22 October 2014 Accepted: 23 February 2015

Published online: 15 March 2015

\section{References}

1. Orditura M, Galizia G, Sforza V, Gambardella V, Fabozzi A, Laterza MM, et al. Treatment of gastric cancer. World J Gastroenterol. 2014;20:1635-49.

2. Kwon HC, Kim SH, Oh SY, Lee S, Lee JH, Jang JS, et al. Clinicopathologic significance of expression of nuclear factor-kappaB RelA and its target gene products in gastric cancer patients. World J Gastroenterol. 2012;18:4744-50.

3. Nashimoto A, Akazawa K, Isobe Y, Miyashiro I, Katai H, Kodera Y, et al. Gastric cancer treated in 2002 in Japan: 2009 annual report of the JGCA nationwide registry. Gastric Cancer. 2013;16:1-27.

4. Schwarz RE, Zagala-Nevarez K. Recurrence patterns after radical gastrectomy for gastric cancer: prognostic factors and implications for postoperative adjuvant therapy. Ann Surg Oncol. 2002;9:394-400.

5. Wagner AD, Grothe W, Haerting J, Kleber G, Grothey A, Fleig WE. Chemotherapy in advanced gastric cancer: a systematic review and meta-analysis based on aggregate data. J Clin Oncol. 2006;24:2903-9.

6. Chau I, Norman AR, Cunningham D, Waters JS, Oates J, Ross PJ. Multivariate prognostic factor analysis in locally advanced and metastatic esophagogastric cancer-pooled analysis from three multicenter, randomized, controlled trials using individual patient data. J Clin Oncol. 2004;22:2395-403.

7. Coussens LM, Werb Z. Inflammation and cancer. Nature. 2002;420:860-7.

8. Gilmour J. Clinical Aspects of Carcinoma of Stomach in Diagnosis - Based Upon the Study of 146 Cases. Br Med J. 1958;1:745-8.

9. Gilmour J. The Surgical Aspects of Gastritis and Its Role in Gastric Pathology. Br J Surg. 1961;49:278-88.

10. Correa P. Clinical Implications of Recent Developments in Gastric-Cancer Pathology and Epidemiology. Semin Oncol. 1985;12:2-10.

11. Ohno S, Inagawa H, Dhar DK, Fujii T, Ueda S, Trchibana M, et al. The degree of macrophage infiltration into the cancer cell nest is a significant predictor of survival in gastric cancer patients. Anticancer Res. 2003;23:5015-22.

12. Qian BZ, Pollard JW. Macrophage Diversity Enhances Tumor Progression and Metastasis. Cell. 2010;141:39-51.

13. Ohta M, Kitadai Y, Tanaka S, Yoshihara M, Yasui W, Mukaida N, et al. Monocyte chemoattractant protein-1 expression correlates with macrophage infiltration and tumor vascularity in human gastric carcinomas. Int J Oncol. 2003;22:773-8.

14. Ishigami S, Natsugoe S, Tokuda K, Nakajo A, Okumura H, Matsumoto M, et al. Tumor-associated macrophage (TAM) infiltration in gastric cancer. Anticancer Res. 2003;23:4079-83.

15. Rudland PS, Platt-Higgins A, El-Tanani M, De Silva Rudland S, Barraclough R, Winstanley JH, et al. Prognostic significance of the metastasis-associated protein osteopontin in human breast cancer. Cancer Res. 2002;62:3417-27.

16. Hu Z, Lin D, Yuan J, Xiao T, Zhang H, Sun W, et al. Overexpression of osteopontin is associated with more aggressive phenotypes in human non-small cell lung cancer. Clin Cancer Res. 2005;11:4646-52.

17. Pan HW, Ou YH, Peng SY, Liu SH, Lai PL, Lee PH, et al. Overexpression of osteopontin is associated with intrahepatic metastasis, early recurrence, and poorer prognosis of surgically resected hepatocellular carcinoma. Cancer. 2003;98:119-27.

18. Wu CY, Wu MS, Chiang EP, Wu CC, Chen YJ, Chen CJ, et al. Elevated plasma osteopontin associated with gastric cancer development, invasion and survival. Gut. 2007;56:782-9. 
19. Agrawal D, Chen T, Irby R, Quackenbush J, Chambers AF, Szabo M, et al. Osteopontin identified as lead marker of colon cancer progression, using pooled sample expression profiling. J Natl Cancer Inst. 2002;94:513-21.

20. Sakaguchi H, Fujimoto J, Hong BL, Tamaya T. Clinical implications of osteopontin in metastatic lesions of uterine cervical cancers. Cancer Lett. 2007;247:98-102.

21. Wang ZM, Cui YH, Li W, Chen YS, Liu TS. Lentiviral-mediated siRNA targeted against osteopontin suppresses the growth and metastasis of gastric cancer cells. Oncol Rep. 2011;25:997-1003.

22. Liu J, Liu Q, Wan Y, Zhao Z, Yu H, Luo H, et al. Osteopontin promotes the progression of gastric cancer through the NF-kappaB pathway regulated by the MAPK and PI3K. Int J Oncol. 2014;45:282-90.

23. Kurahara H, Shinchi H, Mataki Y, Maemura K, Noma H, Kubo F, et al. Significance of M2-polarized tumor-associated macrophage in pancreatic cancer. J Surg Res. 2011;167:e211-9.

24. Mahmoud SM, Lee AH, Paish EC, Macmillan RD, Ellis IO, Green AR. Tumourinfiltrating macrophages and clinical outcome in breast cancer. J Clin Pathol. 2012;65:159-63.

25. Ryder M, Ghossein RA, Ricarte JCM, Knauf JA, Fagin JA. Increased density of tumor-associated macrophages is associated with decreased survival in advanced thyroid cancer. Endocr Relat Cancer. 2008;15:1069-74.

26. Laoui D, Movahedi K, Van Overmeire E, Van den Bossche J, Schouppe E, Mommer C, et al. Tumor-associated macrophages in breast cancer: distinct subsets, distinct functions. Int J Dev Biol. 2011;55:861-7.

27. Mielgo A, Schmid MC. Impact of tumour associated macrophages in pancreatic cancer. BMB Rep. 2013;46:131-8.

28. Imano M, Satou T, Itoh T, Sakai K, Ishimaru E, Yasuda A, et al. Immunohistochemical expression of osteopontin in gastric cancer. J Gastrointest Surg. 2009;13:1577-82.

29. Zhu W, Guo L, Zhang B, Lou L, Lin Z, Zhu X, et al. Combination of osteopontin with peritumoral infiltrating macrophages is associated with poor prognosis of early-stage hepatocellular carcinoma after curative resection. Ann Surg Oncol. 2014;21:1304-13.

30. Kale S, Raja R, Thorat D, Soundararajan G, Patil TV, Kundu GC, et al. Osteopontin signaling upregulates cyclooxygenase-2 expression in tumorassociated macrophages leading to enhanced angiogenesis and melanoma growth via alpha9beta1 integrin. Oncogene. 2014;33:2295-306.

31. Giachelli CM, Lombardi D, Johnson RJ, Murry CE, Almeida M. Evidence for a role of osteopontin in macrophage infiltration in response to pathological stimuli in vivo. Am J Pathol. 1998;152:353-8.

32. Zhou Y, Dai DL, Martinka M, Su M, Zhang Y, Campos El, et al. Osteopontin expression correlates with melanoma invasion. J Invest Dermatol. 2005;124:1044-52.

33. Cheng J, Huo DH, Kuang DM, Yang J, Zheng L, Zhuang SM. Human macrophages promote the motility and invasiveness of osteopontin-knockdown tumor cells. Cancer Res. 2007:67:5141-7.

34. Song G, Ouyang G, Mao Y, Ming Y, Bao S, Hu T. Osteopontin promotes gastric cancer metastasis by augmenting cell survival and invasion through Akt-mediated HIF-1alpha up-regulation and MMP9 activation. J Cell Mol Med. 2009;13:1706-18.

35. Wai PY, Kuo PC. Osteopontin: regulation in tumor metastasis. Cancer Metastasis Rev. 2008;27:103-18.

36. Shevde LA, Samant RS, Paik JC, Metge BJ, Chambers AF, Casey G, et al. Osteopontin knockdown suppresses tumorigenicity of human metastatic breast carcinoma, MDA-MB-435. Clin Exp Metastasis. 2006;23:123-33.

37. Crawford HC, Matrisian LM, Liaw L. Distinct roles of osteopontin in host defense activity and tumor survival during squamous cell carcinoma progression in vivo. Cancer Res. 1998;58:5206-15.

38. Rittling SR, Chambers AF. Role of osteopontin in tumour progression. Br J Cancer. 2004;90:1877-81.

\section{Submit your next manuscript to BioMed Central and take full advantage of:}

- Convenient online submission

- Thorough peer review

- No space constraints or color figure charges

- Immediate publication on acceptance

- Inclusion in PubMed, CAS, Scopus and Google Scholar

- Research which is freely available for redistribution 